\title{
Status of a Supersymmetric Flavour Violating Solution to the Solar Neutrino Puzzle with Three Generations
}

\author{
H. K. Dreiner ${ }^{1 *}$, G. Moreau ${ }^{1,2 \dagger}$ \\ 1: Physikalisches Institut, Nussallee 12 \\ D-53115 Bonn, Germany \\ 2: Service de Physique Théorique \\ CP225, Université Libre de Bruxelles, Bld. du Triomphe \\ 1050 Brussels, Belgium
}

November 16, 2018

\begin{abstract}
We present a general study of a three neutrino flavour transition model based on the supersymmetric interactions which violate R-parity. These interactions induce flavour violating scattering reactions between solar matter and neutrinos. The model does not contain any vacuum mass or mixing angle for the first generation neutrino. Instead, the effective mixing in the first generation is induced via the new interactions. The model provides a natural interpretation of the atmospheric neutrino anomaly, and is consistent with reactor experiments. We determine all R-parity violating couplings which can contribute to the effective neutrino oscillations, and summarize the present laboratory bounds. Independent of the specific nature of the (supersymmetric) flavour violating model, the experimental data on the solar neutrino rates and the recoil electron energy spectrum are inconsistent with the theoretical predictions. The confidence level of the $\chi^{2}$-analysis ranges between $\sim 10^{-4}$ and $\sim 10^{-3}$. The incompatibility, is due to the new SNO results, and excludes the present model. We conclude that a non-vanishing vacuum mixing angle for the first generation neutrino is necessary in our model. We expect this also to apply to the solutions based on other flavour violating interactions having constraints of the same order of magnitude.
\end{abstract}

PACS numbers: 12.60.Jv, 13.15.+g, 14.60.Pq, 14.80.Ly, 26.65.+t

\section{Introduction}

The most elegant solution of the solar neutrino problem [1] is the matter-enhanced neutrino oscillation [2]-[6] (for review articles see [7, 8]). It is based on the Mikheyev-SmirnovWolfenstein (MSW) resonance mechanism [3, 9] which requires neutrino mixing in vacuum.

An attractive alternative interpretation of the solar neutrino deficit is the neutrino flavour transition, due to the existence of some flavour changing (FC) and non-universal flavour diagonal (NFD) interactions between neutrinos and solar matter [9]-[11]. In such a scenario, neutrino oscillations are induced by solar matter alone. Thus the neutrinos can even be massless and no neutrino mixing in vacuum is needed.

\footnotetext{
*e-mail: dreiner@th.physik.uni-bonn.de

$\dagger$ e-mail: moreau@th.physik. uni-bonn.de
} 
The explanation of the solar neutrino puzzle in terms of FC and NFD scattering of neutrinos on solar matter, has been explored quantitatively in [12]-[15], and in [16] for the particular case of neutrinos scattering on electrons characterized by the absence of a resonance. In all these studies, the predicted neutrino rates are consistent with the results from the solar neutrino experiments (Homestake, GALLEX, GNO, SAGE, SNO, Kamiokande and Super-Kamiokande). Acceptable fits have also been found in the framework where both matter-induced neutrino flavour transitions and neutrino mixing in vacuum exist $[12,17]$.

There are also three flavour transition models, solving both the solar and atmospheric neutrino anomalies and in which FC and NFD interactions play a fundamental rôle. In $[18,19]$ a model is constructed in which FC and NFD interactions exist between all neutrino flavours, all the vacuum mixing angles vanish and only the third neutrino is massive (in vacuum). The authors of [20] have considered a scenario where FC and NFD interactions are present between the first and third neutrino flavours, only the vacuum mixing angle of $\nu_{\mu}-\nu_{\tau}$ sector has a significant value and the second and third generation neutrinos are massive (in vacuum). These scenarios have been chosen so that there is a vacuum contribution to the mixing in the $\nu_{\mu}-\nu_{\tau}$ sector because the atmospheric neutrino problem cannot be entirely solved by FC and NFD interactions. The laboratory bounds on the interactions are too severe [21]. The other main constraint, which these models satisfied, is the limit on the $\nu_{e}-\nu_{\tau}$ mixing angle from reactor experiments $[22,23,24]$.

In the present paper, we study the three flavour transition model where FC and NFD interactions are present between all neutrino flavours. However, we restrict the vacuum neutrino masses and a mixing angle to the second and third generation. We thus address the question: do we need a vacuum neutrino mixing angle between the first and second generation to explain the solar neutrino problem or do FC and NFD interactions suffice? The model we study is the same model as in [20] but with the most general FC and NFD interactions.

We concentrate on the situation where the FC and NFD interactions are due to supersymmetry [27] with broken R-parity [25, 26], although our analysis is sufficiently general, that it can be easily applied to any new FC and NFD interaction which is of the same (small) order of magnitude. The $R_{p}$ superpotential is written in terms of the left-handed superfields for the leptons $(L)$, quarks $(Q)$ and Higgs of hypercharge 1/2 $(H)$ and the right-handed superfields for the charged leptons $\left(E^{c}\right)$, up and down type quarks $\left(U^{c}, D^{c}\right)$,

$$
W_{R_{p}}=\sum_{i, j, k}\left(\frac{1}{2} \lambda_{i j k} L_{i} L_{j} E_{k}^{c}+\lambda_{i j k}^{\prime} L_{i} Q_{j} D_{k}^{c}+\frac{1}{2} \lambda_{i j k}^{\prime \prime} U_{i}^{c} D_{j}^{c} D_{k}^{c}+\mu_{i} H L_{i}\right) .
$$

$i, j, k$ are flavour indices, $\lambda_{i j k}, \lambda_{i j k}^{\prime}, \lambda_{i j k}^{\prime \prime}$ are dimensionless coupling constants and $\mu_{i}$ dimension one parameters. We consider all the relevant $R_{p}$ interactions, namely all the different flavour configurations of both $\lambda$ and $\lambda^{\prime}$ couplings, in contrast with the preliminary study made in $[20]$ where only a small set of the $\lambda$ couplings were considered.

Based on the most recent Standard Solar Model (SSM): "BP2000" [28] (and including the new measurement of $S_{17}(0)[29,30]$ ), we determine whether our generic model remains a possible interpretation to the solar neutrino anomaly. We have included the results from the Sudbury Neutrino Observatory (SNO) [31, 32, 33, 34]. In this sense, our work constitutes an update of the preliminary analysis in [20] which was performed before the results from SNO appeared. 


\section{Description of the Model}

\subsection{Hamiltonian}

Our model is characterized by the time evolution equation of neutrino flavour eigenstates,

$$
i \frac{d}{d t}\left(\begin{array}{c}
\nu_{e}(t) \\
\nu_{\mu}(t) \\
\nu_{\tau}(t)
\end{array}\right)=H\left(\begin{array}{c}
\nu_{e}(t) \\
\nu_{\mu}(t) \\
\nu_{\tau}(t)
\end{array}\right),
$$

where the Hamiltonian has a vacuum contribution as well as a matter-induced part:

$$
\begin{aligned}
H= & E \times \mathbf{1}_{3 \times 3}+\left(\frac{\tilde{M}^{2}}{2 E}\right)_{\text {vacuum }}+\left(\frac{\tilde{M}^{2}}{2 E}\right)_{\text {matter }} \\
= & E \times \mathbf{1}_{3 \times 3}+\left(\begin{array}{ccc}
0 & 0 & 0 \\
0 & \frac{m_{3}^{2}+m_{2}^{2}}{4 E}-\frac{m_{3}^{2}-m_{2}^{2}}{4 E} \cos 2 \theta_{23}^{v} & \frac{m_{3}^{2}-m_{2}^{2}}{4 E} \sin 2 \theta_{23}^{v} \\
0 & \frac{m_{3}^{2}-m_{2}^{2}}{4 E} \sin 2 \theta_{23}^{v} & \frac{m_{3}^{2}+m_{2}^{2}}{4 E}+\frac{m_{3}^{2}-m_{2}^{2}}{4 E} \cos 2 \theta_{23}^{v}
\end{array}\right) \\
& +\left(\begin{array}{ccc}
R_{11}+A_{1}+A_{2} & R_{12} & R_{13} \\
R_{12} & R_{22}+A_{2} & R_{23} \\
R_{13} & R_{23} & R_{33}+A_{2}
\end{array}\right) .
\end{aligned}
$$

$E$ is the neutrino energy, $\mathbf{1}_{3 \times 3}$ is the $3 \times 3$ identity matrix, $m_{2}^{2}$ and $m_{3}^{2}$ are, respectively, the second and third generation neutrino masses (in vacuum). $\theta_{23}^{v}$ is the vacuum mixing angle in the $\nu_{\mu}-\nu_{\tau}$ sector, $A_{1}$ and $A_{2}$ are the contributions due respectively to the $W^{ \pm}$ and $Z^{0}$ boson exchanges. Note, that we have omitted any first generation contributions to $\left(\tilde{M}^{2} /(2 E)\right)_{\text {vacuum }}$, as discussed in the introduction.

The $R_{i j}$ terms arise from FC (if $R_{i j} \neq 0[i \neq j]$ ) and NFD (if $R_{11} \neq R_{22} \neq R_{33}$ ) interactions predicted by new physics: here R-parity violating supersymmetry. $A_{1}=$ $\sqrt{2} G_{F} n_{e}$ and $A_{2}=-G_{F} n_{N} / \sqrt{2}$ and $G_{F}$ is the Fermi coupling constant. $n_{e}$ is the electron density and $n_{N}$ the neutron density in the sun. Since we concentrate on $R_{p}$ interactions, the $R_{i j}$ terms are given by

$$
\begin{aligned}
R_{i j} & =R_{i j}(\lambda)+R_{i j}\left(\lambda^{\prime}\right) \\
R_{i j}(\lambda) & =\left(\sum_{k \neq i, j} \frac{\lambda_{i k 1} \lambda_{j k 1}}{4 m^{2}\left(\tilde{l}_{k}^{ \pm}\right)} n_{e}\right)+\left(\sum_{l} \frac{\lambda_{i 1 l} \lambda_{j 1 l}}{4 m^{2}\left(\tilde{\ell}_{l}^{ \pm}\right)} n_{e}\right), \\
R_{i j}\left(\lambda^{\prime}\right) & =\sum_{m}\left(\frac{\lambda_{i m 1}^{\prime} \lambda_{j m 1}^{\prime}}{4 m^{2}\left(\tilde{d}_{m}\right)} n_{d}+\frac{\lambda_{i 1 m}^{\prime} \lambda_{j 1 m}^{\prime}}{4 m^{2}\left(\tilde{d}_{m}\right)} n_{d}\right),
\end{aligned}
$$

where $n_{d}$ is the down quark density and $m^{2}\left(\tilde{l}_{k, l}^{ \pm}\right)\left(m^{2}\left(\tilde{d}_{m}\right)\right)$ is the squared mass of the slepton $\tilde{l}_{k, l}^{ \pm}$(squark $\tilde{d}_{m}$ ) exchanged in the scattering reaction $\nu_{i}+e \stackrel{\lambda}{\rightarrow} \nu_{j}+e\left(\nu_{i}+d \stackrel{\lambda^{\prime}}{\rightarrow} \nu_{j}+d\right)$. The scattering reaction also fixes at least one of the generation indices to be 1 . The second term of $R_{i j}(\lambda)$ vanishes if $i=1$ or $j=1$, due to the anti-symmetry of $\lambda_{i j k}$ in the first two indices. In the following, we perform a general analysis with the $R_{i j}$. Given the approximations discussed in Section 2.3, this analysis can be applied to any new FC and NFD interactions. 


\subsection{Diagonalization}

Since the diagonal contributions to the Hamiltonian do not affect the flavour transition mechanism, only the following effective mass squared matrix,

$$
\left(\frac{\tilde{M}^{2}}{2 E}\right)=H-\left(E+A_{1}+A_{2}\right) \times \mathbf{1}_{3 \times 3},
$$

is relevant. It can be diagonalized as,

$$
U^{\dagger}\left(\frac{\tilde{M}^{2}}{2 E}\right) U=\frac{1}{2 E}\left(\begin{array}{ccc}
\tilde{m}_{1}^{2} & 0 & 0 \\
0 & \tilde{m}_{2}^{2} & 0 \\
0 & 0 & \tilde{m}_{3}^{2}
\end{array}\right)
$$

The unitary matrix $U$ is parameterized by the complex phase $\delta$ and the mixing angles $\theta_{12}, \theta_{13}$ and $\theta_{23}$ as,

$$
\begin{gathered}
U \equiv V_{23} \times V_{13} \times V_{12} \equiv\left(\begin{array}{ccc}
1 & 0 & 0 \\
0 & c_{23} & s_{23} \\
0 & -s_{23} & c_{23}
\end{array}\right)\left(\begin{array}{ccc}
c_{13} & 0 & s_{13} e^{-i \delta} \\
0 & 1 & 0 \\
-s_{13} e^{i \delta} & 0 & c_{13}
\end{array}\right)\left(\begin{array}{ccc}
c_{12} & s_{12} & 0 \\
-s_{12} & c_{12} & 0 \\
0 & 0 & 1
\end{array}\right) \\
U=\left(\begin{array}{ccc}
c_{12} c_{13} & s_{12} c_{13} \\
-s_{12} c_{23}-c_{12} s_{23} s_{13} e^{i \delta} & c_{12} c_{23}-s_{12} s_{23} s_{13} e^{i \delta} & s_{23} c_{13} \\
s_{12} s_{23}-c_{12} c_{23} s_{13} e^{i \delta} & -c_{12} s_{23}-s_{12} c_{23} s_{13} e^{i \delta} & c_{23} c_{13}
\end{array}\right) .
\end{gathered}
$$

Here $s_{i j}=\sin \theta_{i j}$ and $c_{i j}=\cos \theta_{i j}$. In the following, we choose the parameters of the Hamiltonian $H$ to be real, so that $\delta=0$ above. First, we rotate the effective mass squared matrix by $V_{23} \times V_{13}$ and obtain in the new basis,

$$
\left(\frac{\tilde{M}^{2}}{2 E}\right)=\left(\begin{array}{ccc}
R_{11} c_{13}^{2}-\alpha \sin 2 \theta_{13}+\Omega_{+} s_{13}^{2} & \beta c_{13} & 0 \\
\beta c_{13} & \Omega_{-} & \beta s_{13} \\
0 & \beta s_{13} & R_{11} s_{13}^{2}+\alpha \sin 2 \theta_{13}+\Omega_{+} c_{13}^{2}
\end{array}\right),
$$

with,

$$
\begin{gathered}
\alpha \equiv R_{12} s_{23}+R_{13} c_{23}, \quad \beta \equiv R_{12} c_{23}-R_{13} s_{23} \\
\Omega_{ \pm} \equiv\left[\frac{m_{3}^{2}+m_{2}^{2}}{4 E}-A_{1}+\frac{R_{33}+R_{22}}{2}\right] \pm\left[\frac{m_{3}^{2}-m_{2}^{2}}{4 E} \cos 2\left(\theta_{23}^{v}-\theta_{23}\right)\right. \\
\left.+\frac{R_{33}-R_{22}}{2} \cos 2 \theta_{23}+R_{23} \sin 2 \theta_{23}\right] .
\end{gathered}
$$

The mixing angles of Eq. (8)-(10) are given in terms of the Hamiltonian parameters (3)

$$
\tan 2 \theta_{23}=\frac{\sin 2 \theta_{23}^{v} \Delta m_{32}^{2} / 2 E+2 R_{23}}{\cos 2 \theta_{23}^{v} \Delta m_{32}^{2} / 2 E+R_{33}-R_{22}}
$$

where $\Delta m_{32}^{2} \equiv m_{3}^{2}-m_{2}^{2}$, and,

$$
\tan 2 \theta_{13}=\frac{2 \alpha}{\Omega_{+}-R_{11}} .
$$

We show at the end of Section 2.3 that, in our framework, the mixing angle of the $\nu_{e}-\nu_{\tau}$ sector has a negligible value

$$
\theta_{13} \approx 0
$$


Therefore, the upper left $2 \times 2$ part of matrix (8) can be readily diagonalized via the mixing angle $\theta_{12}$ defined by,

$$
\tan 2 \theta_{12} \approx \frac{2 \beta c_{13}}{\Omega_{-}-R_{11} c_{13}^{2}+\alpha \sin 2 \theta_{13}-\Omega_{+} s_{13}^{2}} .
$$

Then, the effective mass eigenvalues are,

$$
\begin{aligned}
& \frac{\tilde{m}_{1}^{2}}{2 E} \approx\left(R_{11} c_{13}^{2}-\alpha \sin 2 \theta_{13}+\Omega_{+} s_{13}^{2}\right) c_{12}^{2}-\beta c_{13} \sin 2 \theta_{12}+\Omega_{-} s_{12}^{2}, \\
& \frac{\tilde{m}_{2}^{2}}{2 E} \approx\left(R_{11} c_{13}^{2}-\alpha \sin 2 \theta_{13}+\Omega_{+} s_{13}^{2}\right) s_{12}^{2}+\beta c_{13} \sin 2 \theta_{12}+\Omega_{-} c_{12}^{2}, \\
& \frac{\tilde{m}_{3}^{2}}{2 E} \approx R_{11} s_{13}^{2}+\alpha \sin 2 \theta_{13}+\Omega_{+} c_{13}^{2} .
\end{aligned}
$$

At this stage, an important comment must be made concerning the $\nu_{e}-\nu_{\mu}$ mixing angle $\theta_{12}$. We see from Eqs. (9) and (14) that $\tan 2 \theta_{12}$ is proportional to the sum of two terms $R_{12} c_{23}$ and $-R_{13} s_{23}$. $R_{12}$ constitutes the off diagonal contribution to the $\nu_{e}-\nu_{\mu}$ sector of the Hamiltonian in Eq. (3). The second term results from a transmission (due to $R_{13}$ ) of the mixing in the $\nu_{\mu}-\nu_{\tau}$ sector (defined by the angle $\theta_{23}$ ) into the $\nu_{e}-\nu_{\mu}$ sector. We conclude that the $R_{12}$ and $R_{13}$ contributions, induced by $R_{p}$ interactions, generate an effective mixing in the $\nu_{e}-\nu_{\mu}$ sector and thus play a fundamental rôle in the neutrino flavour transition mechanism.

\subsection{Approximations and Constraints from Reactor and Atmospheric Neu- trino Experiments}

The less stringent bounds on the products of $R_{p}$ coupling constants are typically of order [35]-[39],

$$
\Lambda \cdot \bar{\Lambda} \lesssim 10^{-2}\left(\frac{m_{\tilde{f}}}{100 \mathrm{GeV}}\right)^{2}, \quad \Lambda \epsilon\left\{\lambda, \lambda^{\prime}, \lambda^{\prime \prime}\right\}
$$

where $m_{\tilde{f}}$ represents the typical scalar superpartner mass. Hence, $R_{i j}$ is at most of order (c.f. Section 3.3 for a more precise discussion):

$$
R_{i j} \lesssim n_{x} \times 2.5 \cdot 10^{-7} \mathrm{GeV}^{-2}, \quad n_{x} \epsilon\left\{n_{e}, n_{d}\right\}
$$

Moreover, inside the sun, $n_{e} \approx n_{d}$, the maximal electron density reached is $n_{e}^{\max } \approx 4.6$. $10^{11} \mathrm{eV}^{3}[28]$ and the energy of the produced neutrinos is never higher than $E=20 \mathrm{MeV}$. Therefore,

$$
\frac{R_{i j}}{\Delta m_{32}^{2} / 2 E}<\mathcal{O}\left(10^{-3}\right)
$$

since $\Delta m_{32}^{2} \approx 10^{-3} \mathrm{eV}^{2}$, as we will see in Eq. (27). In the following, we perform an analysis of the Hamiltonian (3) with general entries $R_{i j}$, however with the restriction (20), above. Thus our analysis applies to any new physics contributing to the $R_{i j}$, which respects the bound (20), i.e. with couplings obeying the bounds (19).

We deduce from Eq. (13) that the experimental data on atmospheric neutrinos [40, 41] are consistent with the $\nu_{\mu} \rightarrow \nu_{\tau}$ oscillation scenario based on an effective two flavour mass matrix, for the following values,

$$
\Delta \tilde{m}_{32}^{2} \equiv \tilde{m}_{3}^{2}-\tilde{m}_{2}^{2} \approx 10^{-3} \mathrm{eV}^{2},
$$


and,

$$
\theta_{23} \approx \frac{\pi}{4} \text {. }
$$

Eqs. (11), (20) and (22) require for the vacuum mixing angle $\theta_{23}^{v}$,

$$
\theta_{23}^{v} \approx \frac{\pi}{4} \approx \theta_{23} .
$$

We also see from Eq. (19) that the ratio of $R_{i j}$ and $A_{1}$ reaches at most the value,

$$
\frac{R_{i j}}{A_{1}} \lesssim 10^{-2}
$$

From Eqs. (13), (14), (23) and (24), it is clear that the mixing angle $\theta_{12}$ is given to a good approximation as,

$$
\tan 2 \theta_{12} \approx \frac{\sqrt{2}\left(R_{12}-R_{13}\right)}{\frac{m_{2}^{2}}{2 E}-A_{1}} .
$$

From Eq. (25) we see there is potentially a resonant point at $\theta_{12}=\pi / 4$. We find that the associated resonance condition, namely $m_{2}^{2} / 2 E=A_{1}$, can be indeed satisfied inside the sun. However, for the atmospheric neutrinos, which have energies of $\mathcal{O}(\mathrm{GeV})$, this resonance condition cannot be fulfilled. The relevant parameter values are: $m_{2}^{2} \approx 5$. $10^{-6} \mathrm{eV}^{2}$ (see Section 3.2) and the electron density inside the earth $n_{e}^{E} \approx(3-6) \mathrm{cm}^{-3} \mathcal{N}_{A}$, where $\mathcal{N}_{A}$ is Avogadro's constant.

From Eqs. (13), (16), (17), (20) and (23), we derive the following approximation for the effective mass squared difference $\Delta \tilde{m}_{32}^{2}$ (c.f. Eq. (21)),

$$
\Delta \tilde{m}_{32}^{2} \approx \Delta m_{32}^{2} \text {. }
$$

We then deduce from Eqs. (21) and (26) that,

$$
\Delta m_{32}^{2} \approx 10^{-3} \mathrm{eV}^{2} \text {. }
$$

Besides, Eqs. (13), (15), (16), (23), (24) and (25) imply that,

$$
\Delta \tilde{m}_{21}^{2}=\tilde{m}_{2}^{2}-\tilde{m}_{1}^{2} \approx m_{2}^{2}-2 E A_{1} .
$$

Finally, we justify the approximation of Eq. (13), used both in the present section and in Section 2.2. First, from Eqs. (23), (24) and (12), we obtain a good approximation for $\theta_{13}$ :

$$
\tan 2 \theta_{13} \approx \frac{\sqrt{2}\left(R_{12}+R_{13}\right)}{\frac{m_{3}^{2}}{2 E}-A_{1}} .
$$

Now, we see from this result and Eq. (24) that $\theta_{13} \approx 0$ outside of the resonance. The resonance point associated with $\theta_{13}$ cannot be reached inside the sun, because the resonance condition $m_{3}^{2} / 2 E=A_{1}$ cannot be satisfied. Indeed, inside the sun (c.f. Eq. (27)),

$$
\frac{m_{3}^{2}}{2 E}>\frac{\Delta m_{32}^{2}}{2 E} \gtrsim 2.5 \cdot 10^{-11} \mathrm{eV}, \quad \text { and, } \quad A_{1} \lesssim 7.510^{-12} \mathrm{eV} .
$$

The resonance condition $m_{3}^{2} / 2 E=A_{1}$ can also not be fulfilled for atmospheric neutrinos, given $n_{e}^{E}$ above. Moreover, it is clear from Eq. (29) that in vacuum, the $\theta_{13}$ mixing angle is exactly equal to zero (like $\theta_{12}$ ), consistent with the constraint obtained at reactor experiments (with an effective two flavour mass matrix): $\sin ^{2} 2 \theta_{13} \lesssim 0.1$ for $\Delta m_{31}^{2} \gtrsim$ $3 \cdot 10^{-3} \mathrm{eV}^{2}[22,23,24]$. Therefore, within the present framework, $\theta_{13}$ approximately vanishes from the point of view of the reactor, solar and atmospheric neutrino experiments. 


\section{Solar Neutrino Rates}

\subsection{Theoretical Transition Probabilities}

Since to a good approximation $\theta_{13}$ is vanishing, the $\nu_{e} \rightarrow \nu_{\mu}$ transitions of solar neutrinos obey a two flavour transition dynamics described by the upper left $2 \times 2$ block of the matrix in Eq. (8). Thus, in the non-adiabatic transition case, the solar $\nu_{e} \rightarrow \nu_{\mu}$ transition probabilities $\bar{P}_{\nu_{e} \rightarrow \nu_{\mu}}=1-\bar{P}_{\nu_{e} \rightarrow \nu_{e}}$ are defined by $[42,43]$,

$$
\bar{P}_{\nu_{e} \rightarrow \nu_{e}} \simeq \frac{1}{2}\left(1-\left(1-2 P_{x}\right) \cos ^{2} 2 \theta_{12}^{s}\right), \quad\left[P_{x}>\frac{1}{2}\right],
$$

for a neutrino produced above the resonance density, and $\bar{P}_{\nu_{e} \rightarrow \nu_{e}} \simeq 1$, for a neutrino produced below the resonance density. In the adiabatic transition case [6],

$$
\bar{P}_{\nu_{e} \rightarrow \nu_{e}} \simeq \frac{1}{2}\left(1+\left(1-2 P_{x}\right) \cos 2 \theta_{12}^{p} \cos 2 \theta_{12}^{s}\right), \quad\left[P_{x}<\frac{1}{2}\right] .
$$

In Eq. (31) and Eq. (32), $\theta_{12}^{p}$ and $\theta_{12}^{s}$ represent the matter-induced $\theta_{12}$ mixing angle at the neutrino production point and solar surface, respectively. $P_{x}$ is the Landau-ZenerStueckelberg transition probability [44],

$$
P_{x} \simeq e^{-\frac{\pi}{2} \gamma F} .
$$

In this equation, $F \simeq 1-\tan ^{2} \theta_{12}^{v a c}[8]$, and $\theta_{12}^{v a c}$ is the $\theta_{12}$ mixing angle in vacuum, since the electron density inside the sun is approximately given by an exponential function of the distance $r$ to the solar center [28]. From Eq. (25) we deduce that $\theta_{12}^{\text {vac }} \approx 0$, so that $F \simeq 1$ here. The $\gamma$ parameter of Eq. (33) is given at the resonance point,

$$
\gamma \equiv\left|\frac{\Delta \tilde{m}_{21}^{2} / 2 E}{2 \frac{d \theta_{12}}{d r}}\right|_{r e s} .
$$

The resonance point is determined by (c.f. Eq. (25)),

$$
\frac{m_{2}^{2}}{2 E}=A_{1} \text {. }
$$

Let us derive the expression for $\gamma$ within our model. At the resonance point, $\theta_{12}=\pi / 4$ so that Eqs. (15) and (16), together with Eqs. (13) and (23), give,

$$
\left|\frac{\Delta \tilde{m}_{21}^{2}}{2 E}\right|_{\text {res }}=\sqrt{2}\left|R_{12}-R_{13}\right|_{\text {res }} .
$$

From Eq. (25) we see that $\left|2 d \theta_{12} / d r\right|_{\text {res }}=G_{F}\left|\frac{d n_{e}}{d r} /\left(R_{12}-R_{13}\right)\right|_{\text {res }}$ and thus,

$$
\gamma=\frac{\sqrt{2}\left|R_{12}-R_{13}\right|_{r e s}^{2}}{G_{F}\left|\frac{d n_{e}}{d r}\right|_{r e s}}
$$

\subsection{Fit with Experimental Results}

In this subsection, we present the free parameter values giving rise to the highest degree of consistency between our theoretical predictions and the experimental solar neutrino rates. The relevant free parameters in Eqs. $(31,32)$ are $m_{2}^{2}$ and $R$, where

$$
R \equiv \frac{\left|R_{12}(\lambda)-R_{13}(\lambda)\right|}{n_{e}}
$$


for $\lambda-R_{p}$ interactions and

$$
R \equiv \frac{\left|R_{12}\left(\lambda^{\prime}\right)-R_{13}\left(\lambda^{\prime}\right)\right|}{n_{d}},
$$

for $\lambda^{\prime}-R_{p}$ interactions (c.f. Eq. (4)). In certain specified cases, we also consider $f_{B}$ as a free parameter: $f_{B}$ is defined by $\Phi_{8_{B}}=f_{B} \times \Phi_{8_{B}}^{S S M}$, where $\Phi_{8_{B}}$ is the flux of solar electronneutrinos produced in the decay ${ }^{8} B \rightarrow{ }^{8} B e^{*}+e^{+}+\nu_{e}$ and $\Phi_{8_{B}}^{S S M}$ is the theoretical prediction within the $\operatorname{SSM}[28,29,30]$. This is equivalent to allowing for an arbitrary normalization of the $\Phi_{8_{B}}$ flux.

In Table 1, we show the values of the free parameters for which the best fit is obtained between the theoretical solar neutrino rates defined as,

$$
P_{j}^{t h}=\sum_{i} \phi_{i} \int d E d r \frac{d g_{i}(E)}{d E} \frac{d h_{i}(r)}{d r} \sigma_{j}(E) \bar{P}_{\nu_{e} \rightarrow \nu_{e}}(E, r),
$$

and the corresponding present experimental solar neutrino rates $P_{j}^{\exp }$ [31]-[34], [45]-[50]. The index $j$ runs over the 5 solar neutrino experiments (we use the combined result for the GALLEX, GNO and SAGE experiments [48]): $j=\{$ Homestake, GALLEX + GNO + SAGE, SNO, Kamiokande, Super-Kamiokande\}.

Let us briefly describe the parameters entering Eq. (40). First, $P_{j}^{\text {th }}$ is the theoretical prediction for the solar neutrino detection rate in experiment $j$, if neutrinos have a flavour transition average probability $\bar{P}_{\nu_{e} \rightarrow \nu_{e}}(E, r)$. The $\phi_{i}$ are the flux of solar electron-neutrinos, where $i$ labels the source reaction: $i=\left\{\mathrm{pp},{ }^{7} \mathrm{Be}\right.$, pep, ${ }^{13} \mathrm{~N},{ }^{15} \mathrm{O},{ }^{17} \mathrm{~F},{ }^{8} \mathrm{~B}$, hep $\} . d g_{i}(E) / d E$ and $d h_{i}(r) / d r$ respectively represent the neutrino energy and the initial radial position distributions (normalized to unity). Both these fluxes and distributions are taken from the most recent SSM (BP2000) [28]-[30]. Finally, the $\sigma_{j}(E)$ denote the neutrino detection cross sections corresponding to the different solar neutrino experiments $[1,51,52,53]$.

In the case of the Kamiokande and Super-Kamiokande experiments, the product $\sigma_{j}(E)$ $\bar{P}_{\nu_{e} \rightarrow \nu_{e}}(E, r)$ in Eq. (40) stands for,

$$
\begin{array}{r}
\int_{T_{t h}}^{T_{\max }} d T \int_{0}^{\infty} d T^{\prime} \epsilon\left(T^{\prime}\right) \rho\left(T, T^{\prime}\right)\left[\frac{d \sigma_{j}^{\nu_{e}}\left(E, T^{\prime}\right)}{d T^{\prime}} \bar{P}_{\nu_{e} \rightarrow \nu_{e}}(E, r)\right. \\
\left.+\frac{d \sigma_{j}^{\nu_{\mu}}\left(E, T^{\prime}\right)}{d T^{\prime}}\left(1-\bar{P}_{\nu_{e} \rightarrow \nu_{e}}(E, r)\right)\right],
\end{array}
$$

where $T^{\prime}$ is the true energy of the recoil electron, $T$ its value measured experimentally, $T_{t h}$ the energy threshold of the experiment, $T_{\max }$ the maximal energy of the experiment, $\epsilon\left(T^{\prime}\right)$ the detection efficiency, $\rho\left(T, T^{\prime}\right)$ the energy resolution function and $d \sigma_{j}^{\nu_{e}}\left(E, T^{\prime}\right) / d T^{\prime}$ $\left(d \sigma_{j}^{\nu_{\mu}}\left(E, T^{\prime}\right) / d T^{\prime}\right)$ the differential cross section for the solar electron-neutrino (muonneutrino) elastic scattering reaction on electrons [1]. The resolution function of Eq. (41) can be satisfactorily represented by the following Gaussian function [54],

$$
\rho\left(T, T^{\prime}\right)=\frac{e^{\frac{-\left(T-T^{\prime}\right)^{2}}{2 \Delta^{2}\left(T^{\prime}\right)}}}{\sqrt{2 \pi \Delta^{2}\left(T^{\prime}\right)}} .
$$

The standard deviation of the energy measurement is approximately given by,

$$
\frac{\Delta\left(T^{\prime}\right)}{T^{\prime}} \approx a\left(\frac{10 M e V}{T^{\prime}}\right)^{1 / 2}
$$

We take $T_{t h}=7.5 \mathrm{MeV}$ [49], $T_{\max }=20 \mathrm{MeV}[49], \epsilon \approx 0.7[1]$ and $a=0.143[49,55,56,57]$, for the Kamiokande detector, and $T_{t h}=5 \mathrm{MeV}[50], T_{\max }=20 \mathrm{MeV}[50], \epsilon \approx 0.7[50,58]$ and $a=0.153[59,60]$, for the Super-Kamiokande one. 
For the SNO experiment, the product $\sigma_{j}(E) \bar{P}_{\nu_{e} \rightarrow \nu_{e}}(E, r)$ in Eq. (40) stands for,

$$
\int_{T_{t h}^{S N O}}^{T_{\max }^{S N O}} d T \int_{0}^{\infty} d T^{\prime} \rho\left(T, T^{\prime}\right) \frac{d \sigma_{j}^{C C}\left(E, T^{\prime}\right)}{d T^{\prime}} \bar{P}_{\nu_{e} \rightarrow \nu_{e}}(E, r) .
$$

$d \sigma_{j}^{C C}\left(E, T^{\prime}\right) / d T^{\prime}$ is the differential cross section of the charged current reaction between the solar electron-neutrinos and deuterium: $\nu_{e}+D \rightarrow p+p+e^{-}$[53]. The energy resolution function $\rho\left(T, T^{\prime}\right)$ of Eq. (44) is defined as before by Eq. (42), but with the following standard deviation $\Delta\left(T^{\prime}\right)[31]$,

$$
\Delta\left(T^{\prime}\right)=\left[-0.4620+0.5470 \sqrt{T^{\prime} / 1 \mathrm{MeV}}+0.008722\left(T^{\prime} / 1 \mathrm{MeV}\right)\right] \mathrm{MeV} .
$$

In the present analysis, we use $T_{t h}^{S N O}=6.75 \mathrm{MeV}+m_{e}$ [31], $m_{e}$ is the electron mass, and $T_{\max }^{S N O}=13 \mathrm{MeV}+m_{e}[31]$.

The points of parameter space corresponding to the best fit between the theoretical and experimental solar neutrino rates have been obtained by performing a $\chi^{2}$-analysis. Following the analyses of $[61,62]$, we define our $\chi^{2}$-function as,

$$
\chi_{R}^{2}=\sum_{j_{1}, j_{2}}\left(P_{j_{1}}^{t h}-P_{j_{1}}^{e x p}\right)\left[\sigma_{R}^{2}\right]_{j_{1} j_{2}}^{-1}\left(P_{j_{2}}^{t h}-P_{j_{2}}^{e x p}\right),
$$

where $j_{1}$ and $j_{2}$ run over the solar neutrino experiments and $\left[\sigma_{R}^{2}\right]_{j_{1} j_{2}}$ is the squared error matrix containing both the experimental (systematic and statistical) [31, 32, 45], [48][50] and theoretical errors (computed according to the analyses of [63, 64]) on the solar neutrino rates. The theoretical errors depend on the uncertainties of both the neutrino fluxes [28, 30], [65]-[68] and the detection cross sections [51]-[53], [69].

In Table 1, our results concerning the fits of solar neutrino rates are given in two different contexts: before (case (1)) and after the publication of experimental data from SNO [31]-[34] (case (2)). In the "post-SNO" context, we include into our $\chi^{2}$-analysis the rate of solar electron-neutrinos detected at the SNO experiment via the charged current reaction on deuterium [31]. Furthermore, within this context, we fix the normalization factor $f_{B}$ to unity, following the study of [15]. The reason is that the value of the neutrino flux $\Phi_{8_{B}}$ measured at the SNO experiment (with the flux of neutrinos detected through the neutral current reaction on deuterium) $[31,32,34]$ is in agreement with its prediction in the $\operatorname{SSM}[28,29,30]: \Phi_{8_{B}}^{S S M}$. Finally, let us note that within this post-SNO context, we do not implement into the $\chi^{2}$-analysis the rate of solar neutrinos detected at the SNO experiment via the elastic scattering reaction on electrons [31, 32, 34]. This is justified by the fact that this rate is consistent with the rates of neutrinos detected at the Kamiokande and Super-Kamiokande experiments via the same scattering reaction [49, 50], which are already taken into account in our analysis.

In Table 1, we also present the values of the parameters corresponding to the best fit of all 5 solar neutrino rates and the recoil electron energy spectrum (case (3)). This fit takes into account the consistency between the recoil electron energy spectrum obtained with the Super-Kamiokande detector from 1258 days of data [50] and its theoretical prediction. These results have also been derived through the $\chi^{2}$-method with the $\chi^{2}$-function $[61,62]$,

$$
\chi_{S}^{2}=\chi_{R}^{2}+\sum_{j_{1}, j_{2}}\left(\bar{\alpha} S_{j_{1}}^{t h}-S_{j_{1}}^{e x p}\right)\left[\sigma_{S}^{2}\right]_{j_{1} j_{2}}^{-1}\left(\bar{\alpha} S_{j_{2}}^{t h}-S_{j_{2}}^{e x p}\right)
$$

Here the $S_{j}^{\exp }[j=1, \ldots, 19]$ are the solar neutrino rates observed in the Super-Kamiokande experiment corresponding to the 19 bins of a given recoil electron energy range [50] and 


\begin{tabular}{|c|c|c|c|c|c|c|c|c|c|}
\hline $\begin{array}{c}\text { Model } \\
(\text { fit })\end{array}$ & $\begin{array}{c}m_{2}^{2} \\
\left(10^{-5}\right. \\
\left.\mathrm{eV}^{2}\right)\end{array}$ & $\begin{array}{c}R \\
\left(10^{-24}\right. \\
\left.\mathrm{eV}^{-2}\right)\end{array}$ & $f_{B} / \bar{\alpha}$ & $\begin{array}{c}\chi^{2} \\
(D O F ; C . L .)\end{array}$ & $\begin{array}{c}\mathrm{H} \\
(0.297 \\
\pm 0.026)\end{array}$ & $\begin{array}{c}\mathrm{G}+\mathrm{G}+\mathrm{S} \\
(0.557 \\
\pm 0.039)\end{array}$ & $\begin{array}{c}\mathrm{SNO} \\
(0.295 \\
\pm 0.023)\end{array}$ & $\begin{array}{c}\mathrm{K} \\
(0.472 \\
\pm 0.064)\end{array}$ & $\begin{array}{c}\text { SK } \\
(0.391 \\
\pm 0.014\end{array}$ \\
\hline $\begin{array}{c}\lambda \\
(1)\end{array}$ & 0.545 & 1.319 & $\begin{array}{c}f_{B}= \\
1.087\end{array}$ & $\begin{array}{c}0.19 \\
(1 ; 66.3 \%)\end{array}$ & 0.295 & 0.574 & $\times$ & 0.476 & 0.390 \\
\hline$\lambda$ & 0.625 & 1.055 & & $\begin{array}{c}6.25 \\
(3 ; 10.0 \%)\end{array}$ & 0.244 & 0.595 & 0.316 & 0.402 & 0.369 \\
\hline $\begin{array}{c}\lambda \\
(2)\end{array}$ & 0.648 & 0.561 & $\begin{array}{c}\bar{\alpha}= \\
0.624\end{array}$ & $\begin{array}{c}46.60 \\
(21 ; 0.11 \%)\end{array}$ & 0.560 & 0.645 & 0.704 & 0.738 & 0.713 \\
\hline $\begin{array}{c}\lambda^{\prime} \\
(1)\end{array}$ & 0.534 & 1.112 & $\begin{array}{c}f_{B}= \\
1.115\end{array}$ & $\begin{array}{c}0.18 \\
(1 ; 67.1 \%)\end{array}$ & 0.303 & 0.562 & $\times$ & 0.491 & 0.389 \\
\hline $\begin{array}{c}\lambda^{\prime} \\
(2)\end{array}$ & 0.625 & 0.814 & & $\begin{array}{c}6.23 \\
(3 ; 10.1 \%)\end{array}$ & 0.244 & 0.595 & 0.316 & 0.402 & 0.369 \\
\hline$\lambda^{\prime}$ & 0.386 & 0.257 & $\begin{array}{c}\bar{\alpha}= \\
(3)\end{array}$ & $\begin{array}{c}51.73 \\
(21 ; 0.021 \%)\end{array}$ & 0.865 & 0.629 & 0.997 & 0.992 & 0.972 \\
\hline
\end{tabular}

Table 1: The values of the parameters $m_{2}^{2}, R, f_{B}$ and $\bar{\alpha}$ (see text) associated with the best fits between the solar neutrino experimental data and the theoretical prediction. They are given in the 3 cases we consider: (1) the solar neutrino rates except those of the SNO experiment, (2) all the solar neutrino rates, including the rates detected at the SNO experiment via the charged current reaction, and (3) all the solar neutrino rates together with the recoil electron energy spectrum obtained at the Super-Kamiokande experiment. Both the models based on the $R_{p}$ interactions of type $\lambda$ and $\lambda^{\prime}$ are considered. We show the values, computed with the obtained best-fit parameters, of the relevant $\chi^{2}$-function (with associated number of degrees of freedom denoted as DOF and confidence level denoted as C.L.), namely either $\chi_{R}^{2}$ (for cases 1 and 2) or $\chi_{S}^{2}$ (for case 3). We also indicate, for the best-fit parameters, the theoretical solar neutrino rates $P_{j}^{t h}$ (see Eq. (40)) normalized to their value expected in the SSM BP2000 [28, 29, 30], at the considered experiments: Homestake $(\mathrm{H})$, GALLEX+GNO+SAGE $(\mathrm{G}+\mathrm{G}+\mathrm{S})$, SNO, Kamiokande (K) and Super-Kamiokande (SK). The corresponding present experimental solar neutrino rates $P_{j}^{\text {exp }}$ (together with their experimental uncertainty) [31]-[34], [45]-[50] are written in parentheses. Finally, we note that when the value of $f_{B}$ or $\bar{\alpha}$ (which are presented in the same column) is not given, it means that it is fixed to unity and/or it is not relevant.

the $S_{j}^{t h}[j=1, \ldots, 19]$ are their predicted values. The $S_{j}^{t h}$ are calculated with Eqs. (40)(43) (applied to the Super-Kamiokande experiment) by integrating $T$ over the relevant 19 energy intervals [50]. The matrix $\left[\sigma_{S}^{2}\right]_{j_{1} j_{2}}$ entering Eq. (47) is equal to [14],

$$
\left[\sigma_{S}^{2}\right]_{j_{1} j_{2}}=\delta_{j_{1} j_{2}}\left(\left[\sigma_{\text {stat }}^{2}\right]_{j_{1} j_{2}}+\left[\sigma_{\text {uncorr }}^{2}\right]_{j_{1} j_{2}}\right)+\left[\sigma_{\text {corr }}^{2}\right]_{j_{1} j_{2}}+\left[\sigma_{t h}^{2}\right]_{j_{1} j_{2}} .
$$

The $\left[\sigma_{\text {stat }}^{2}\right]_{j_{1} j_{2}}$ represent the squared experimental statistical errors $[50],\left[\sigma_{\text {corr }}^{2}\right]_{j_{1} j_{2}}$ and $\left[\sigma_{u n c o r r}^{2}\right]_{j_{1} j_{2}}$ are respectively the correlated and uncorrelated contributions to the squared experimental systematic errors $[50]$ and $\left[\sigma_{t h}^{2}\right]_{j_{1} j_{2}}$ are the squared theoretical uncertainties [28, 30], [63]-[68]. Finally, the overall normalization factor $\bar{\alpha}$ introduced in Eq. (47) has been taken as an additional free parameter in the fit, in order to avoid double-counting with the data from the Super-Kamiokande experiment on the total event rate which is already included in $\chi_{R}^{2}$.

Let us comment on the results of the $\chi^{2}$-analyses given in Table 1 . In case one considers the solar neutrino rates excluding the SNO experiment, case (1), one obtains acceptable fits between the theoretical and the experimental rates, namely fits at $66.3 \%$ and $67.1 \%$ of Confidence Level (C.L.) in the scenarios containing $\lambda$ and $\lambda^{\prime}$ interactions, respectively. When adding the SNO results to the analysis, the fit grows significantly worse: the theoretical predictions are now compatible with the experimental data only at the $10.0 \%$ C.L. and $10.1 \%$ C.L., respectively. Finally, considering all the solar neutrino rates (including the SNO data) as well as the recoil electron energy spectrum, case (3), the obtained fits 
have a confidence level of $1.1 \times 10^{-3}$ C.L. and $2.1 \times 10^{-4}$ C.L., respectively: the theoretical and experimental results are not compatible in this case. This incompatibility is due to the SNO results and not the recoil electron energy spectrum. In [20] the authors obtained an acceptable fit, without the SNO data but including the recoil electron energy spectrum.

Furthermore, we observe in Table 1 that the values of the quantity $R$, corresponding to the best fit, are smaller in case the solar neutrino flavour transitions are induced by $R_{p}$ interactions of the type $\lambda^{\prime}$ instead of $\lambda$. The reason is that the quantity (c.f. Eq. (38)),

$$
X_{\lambda}=\left|R_{12}-R_{13}\right|=\left|R_{12}(\lambda)-R_{13}(\lambda)\right|=R n_{e},
$$

entering the transition probability $\bar{P}_{\nu_{e} \rightarrow \nu_{e}}$ (see Section 3.1), in case neutrino flavour transitions are due to $\lambda$ couplings, is replaced by (c.f. Eq. (39)),

$$
X_{\lambda^{\prime}}=\left|R_{12}-R_{13}\right|=\left|R_{12}\left(\lambda^{\prime}\right)-R_{13}\left(\lambda^{\prime}\right)\right|=R n_{d} .
$$

The decisive difference is that $0.50 n_{d} \lesssim n_{e} \lesssim 0.78 n_{d}$ inside the sun $[14,28]$. In other words, a modification of the matter-induced transition probability $\bar{P}_{\nu_{e} \rightarrow \nu_{e}}$ due to an increase of the solar matter density can be compensated by a decrease of effective neutrino $\not R$ interaction strength (quantified here by $R$ ). Let us note that this compensation is not exact, as the solar matter density depends on the radial position, while this is not true for the parameter $R$. This is why, in Table 1, the best-fit values of the other free parameters, $m_{2}^{2}$ and $f_{B}$ (or $\bar{\alpha}$ ), are also different in the two situations where flavour transitions are due to $\lambda$ and $\lambda^{\prime}$ couplings.

Let us make a brief final remark: we have found that, in the scenario where solar neutrino flavour transitions are simultaneously induced by $R_{p}$ interactions of type $\lambda$ and $\lambda^{\prime}$, the fits between observed and predicted solar neutrino rates and recoil electron energy spectrum are not significantly improved compared to the fits for which the results are shown in Table 1.

\subsection{Comparison of the Results with the Bounds on the $R_{p}$ Coupling Con- stants}

In this section, we check that the relevant present limits on $R_{p}$ coupling constants are compatible with the values of $R$ giving rise to the best fits of solar neutrino rates (see previous section). This check is also a self-consistency check. It allows us to verify that the typical bound on the relevant $R_{p}$ coupling constants (19), on which the approximations (see Section 2.3) used in the mass matrix diagonalization are based, are correct.

- $\lambda$ coupling constants: For $\lambda$-couplings $R$ reads explicitly (c.f. Eq. (4)),

$$
R=\left|\frac{\lambda_{131} \lambda_{231}}{4 m^{2}\left(\tilde{\tau}^{ \pm}\right)}-\frac{\lambda_{121} \lambda_{321}}{4 m^{2}\left(\tilde{\mu}^{ \pm}\right)}\right| .
$$

The largest best fit value for $R$ in Table 1 is $1.31910^{-24} \mathrm{eV}^{-2}$. The bounds on the R-parity violating couplings are usually given as bounds on a single coupling or on a product of two couplings. This is based on the assumption that the other couplings vanish ${ }^{1}$. We adopt this approximation in the following.

No bound exists on the $R_{p}$ coupling constant product $\lambda_{121} \lambda_{321}$. The present experimental constraints at $2 \sigma$ on the single $R_{p}$ coupling constants $\lambda_{121}$ and $\lambda_{321}$ are given

\footnotetext{
${ }^{1}$ The assumption that a single or a pair of $\not R_{p}$ coupling constants are dominant is often adopted in the literature, as a simplification. This hypothesis is justified by the analogy between structures of $\not R_{p}$ and Higgs Yukawa interactions [70]. The latter exhibit a strong hierarchy in flavour space.
} 
respectively by $[35,38,39,71]$,

$$
\lambda_{121}<0.049\left(\frac{m\left(\tilde{e}_{R}^{ \pm}\right)}{100 \mathrm{GeV}}\right), \quad \lambda_{321}<0.07\left(\frac{m\left(\tilde{e}_{R}^{ \pm}\right)}{100 \mathrm{GeV}}\right) .
$$

Note that a different slepton mass appears in $R$ and in the bound above. If we assume that $m(\tilde{\mu})=m\left(\tilde{e}_{R}\right)$, we obtain from the second term in Eq. (51) that $R<9 \cdot 10^{-26} \mathrm{eV}^{-2}$ and this model irrelevant for FC and NFD interactions in the sun. If however $m(\tilde{\mu})=$ $m\left(\tilde{e}_{R}\right) / 4$, which could very well be the case with non-universal boundary conditions at the unification scale,

$$
R<1.372 \cdot 10^{-24} \mathrm{eV}^{-2},
$$

and the $\lambda_{121} \lambda_{321}$ interactions can reach the best fit values for $R$.

The strongest bound on the Yukawa coupling constant product $\lambda_{131} \lambda_{231}$ is [72],

$$
\lambda_{131} \lambda_{231}<6.610^{-7}\left(\frac{m\left(\tilde{\nu}_{\tau L}\right)}{100 \mathrm{GeV}}\right)^{2} .
$$

In this case we would need to require $m\left(\tilde{\nu}_{\tau L}\right) \approx 300 \times m(\tilde{\tau})$ in order to obtain a sufficiently large $R$. For $m(\tilde{\tau}) \gtrsim 100 \mathrm{GeV}$ (the LEP bound [73]), this is not compatible with a supersymmetric solution to the hierarchy problem. We conclude that these interactions can not significantly contribute to FC and NFD interactions in the sun.

In summary, only when $R$ is given by $R=\left|\lambda_{121} \lambda_{321}\right| / 4 m^{2}\left(\tilde{\mu}^{ \pm}\right)$, the present constraints on relevant $R_{p}$ interactions allow $R$ to take the relevant value of $1.31910^{-24} \mathrm{eV}^{-2}$.

- $\lambda^{\prime}$ coupling constants: Let us now consider the model (not studied in paper [20]) in which neutrino flavour transitions are due to $\lambda^{\prime} R_{p}$-interactions via solar down quarks.

$$
R=\left|\sum_{m}\left(\frac{\lambda_{1 m 1}^{\prime} \lambda_{2 m 1}^{\prime}}{4 m^{2}\left(\tilde{d}_{m}\right)}+\frac{\lambda_{11 m}^{\prime} \lambda_{21 m}^{\prime}}{4 m^{2}\left(\tilde{d}_{m}\right)}-\frac{\lambda_{1 m 1}^{\prime} \lambda_{3 m 1}^{\prime}}{4 m^{2}\left(\tilde{d}_{m}\right)}-\frac{\lambda_{11 m}^{\prime} \lambda_{31 m}^{\prime}}{4 m^{2}\left(\tilde{d}_{m}\right)}\right)\right| .
$$

Based on the present constraints on $R_{p}$ interactions, we determine whether $R$ can reach $1.111^{-24} \mathrm{eV}^{-2}$, which is the maximal value appearing in Table 1. As before, we consider separately each term of Eq. (55), supposing that it dominates over all the others.

The product $\lambda_{121}^{\prime} \lambda_{321}^{\prime}$ (third term in $R$ with $m=2$ ) is only constrained by the present individual experimental limits at $2 \sigma$ on $\lambda_{121}^{\prime}$ and $\lambda_{321}^{\prime}[38,39,71]$,

$$
\lambda_{121}^{\prime}<0.043\left(\frac{m\left(\tilde{d}_{R}\right)}{100 \mathrm{GeV}}\right), \quad \lambda_{321}^{\prime}<0.52\left(\frac{m\left(\tilde{d}_{R}\right)}{100 \mathrm{GeV}}\right) .
$$

Again a different squark mass appears in $R, m(\tilde{s})$, than in the bounds, $m\left(\tilde{d}_{R}\right)$. If we assume they are equal, then

$$
R<5.6 \cdot 10^{-25} \mathrm{eV}^{-2}
$$

This is just below the maximal best-fit value. For a moderate non-degeneracy of $m\left(\tilde{d}_{R}\right)=$ $1.5 \times m(\tilde{s})$, the maximal best-fit value can be obtained.

The Yukawa coupling constant product $\lambda_{131}^{\prime} \lambda_{331}^{\prime}$ (third term in $R$, with $m=3$ ) is only constrained by the present individual experimental bounds on $\lambda_{131}^{\prime}$ (at $3 \sigma$ ) [39, 71] and on $\lambda_{331}^{\prime}($ at $2 \sigma)[36,39,74]$ :

$$
\lambda_{131}^{\prime}<0.019\left(\frac{m\left(\tilde{t}_{L}\right)}{100 \mathrm{GeV}}\right), \quad \lambda_{331}^{\prime}<0.45 \quad \text { at } m(\tilde{d})=100 \mathrm{GeV} .
$$

Assuming all squark masses are equal

$$
R<2.1 \cdot 10^{-25} \mathrm{eV}^{-2},
$$


which is below the best-fit values in Table 1 . Given a non-degeneracy of $m(\tilde{d})=m\left(\tilde{t}_{L}\right)=$ $2.3 \times m(\tilde{b})$, the fit values for $R$ can be reached.

For $m=1$ we have for the last two terms the coupling $\lambda_{111}^{\prime}$, which has the strict bound at $2 \sigma[39,75,76]$,

$$
\lambda_{111}^{\prime}<5.210^{-4}\left(\frac{m(\tilde{e})}{100 \mathrm{GeV}}\right)^{2}\left(\frac{m\left(\tilde{\chi}^{0}\right)}{100 \mathrm{GeV}}\right)^{1 / 2} .
$$

In these cases, we can therefore not reach the fit values for $R$, unless we require an extreme hierarchy in superpartner masses.

The present experimental bounds at $2 \sigma$ on $\lambda_{312}^{\prime}$ and $\lambda_{112}^{\prime}[38,39,71]$ (fourth term in $R$ with $m=2$ ) are,

$$
\lambda_{312}^{\prime}<0.11\left(\frac{m\left(\tilde{s}_{R}\right)}{100 G e V}\right), \quad \lambda_{112}^{\prime}<0.021\left(\frac{m\left(\tilde{s}_{R}\right)}{100 G e V}\right) .
$$

In this case we always have the same squark mass and we can deduce a rigorous bound

$$
R<5.8 \cdot 10^{-26} \mathrm{eV}^{-2}
$$

which excludes any relevant contribution to FC and NFD interactions in the sun.

In the same way, the present experimental limits at $2 \sigma$ on $\lambda_{313}^{\prime}$ and $\lambda_{113}^{\prime}[38,39,71]$,

$$
\lambda_{313}^{\prime}<0.11\left(\frac{m\left(\tilde{b}_{R}\right)}{100 G e V}\right), \quad \lambda_{113}^{\prime}<0.021\left(\frac{m\left(\tilde{b}_{R}\right)}{100 G e V}\right)
$$

result in $R<5.8 \cdot 10^{-26} \mathrm{eV}^{-2}$, precluding any significant contribution.

The three products of Yukawa coupling constants $\lambda_{11 m}^{\prime} \lambda_{21 m}^{\prime}, m=1,2,3$, (second term in $R$ ) suffer the following strong constraint [78],

$$
\lambda_{11 k}^{\prime} \lambda_{21 k}^{\prime}<1.7 \cdot 10^{-7}\left(\frac{m\left(\tilde{d}_{k R}\right)}{100 G e V}\right)^{2} .
$$

Hence, $R<4.25 \cdot 10^{-30} \mathrm{eV}^{-2}$ and no interesting solutions exist.

The products $\lambda_{1 m 1}^{\prime} \lambda_{2 m 1}^{\prime}, m=1,2,3$ (first term in $R$ ) also have a stringent limit [78]:

$$
\lambda_{1 j 1}^{\prime} \lambda_{2 j 1}^{\prime}<1.610^{-7}\left(\frac{m\left(\tilde{u}_{j L}\right)}{100 G e V}\right)^{2}
$$

precluding any significant contributions to the FC and NFD interactions in the sun.

In summary, if the two coupling constants $\lambda_{121}^{\prime}$ and $\lambda_{321}^{\prime}$, or $\lambda_{131}^{\prime}$ and $\lambda_{331}^{\prime}$, are dominant so that the quantity $R$ is given by $R=\left|\lambda_{121}^{\prime} \lambda_{321}^{\prime}\right| / 4 m^{2}(\tilde{s})$, or $R=\left|\lambda_{131}^{\prime} \lambda_{331}^{\prime}\right| / 4 m^{2}(\tilde{b})$, respectively, the present bounds on relevant $R_{p}$ coupling constants allow $R$ to reach the relevant value: $1.11210^{-24} \mathrm{eV}^{-2}$.

\section{Conclusions}

We have studied a three neutrino flavour transition model (see Section 2.1), based on the supersymmetric $R_{p}$-interactions between neutrinos and solar matter (see Section 2.2). We have not included a vacuum mass or any vacuum mixing angles for the first generation neutrino. Instead the effective mixing is induced via the flavour violating interactions.

Within this scenario, we have found that, among all the $\left(R_{p}\right)$ contributions $R_{i j}$ to the matter-induced part of the effective mass matrix in Eq.(3), only $R_{12}$ and $R_{13}$ play a rôle 
(see Section 2.3). Then we have shown, from a systematic study of the constraints on the $R_{p}$ couplings, that only the products $\lambda_{121} \lambda_{321}, \lambda_{121}^{\prime} \lambda_{321}^{\prime}$ and $\lambda_{131}^{\prime} \lambda_{331}^{\prime}$, which enter $R_{13}$, can result in a sufficiently large value of $R$ in agreement with the best fit obtained for the solar neutrino data (see Section 3.3).

However, whatever the flavour configurations of the $R_{p}$ contributions are, we have found that the best fits of all solar neutrino rates together with the recoil electron energy spectrum have a confidence level ranging between $\sim 10^{-3}$ (scenario with $\lambda$ couplings) and $\sim 10^{-4}$ (scenario with $\lambda^{\prime}$ couplings). The discrepancy between the experimental results and the theoretical predictions is due to the results from the SNO experiment. The considered model, which provides a realistic interpretation of the atmospheric neutrino anomaly and respects the constraints on neutrino oscillations obtained at reactor experiments (see Section 2.3), does not constitute an acceptable solution to the solar neutrino problem. This analysis does not rely on the nature of the $R_{p}$-interactions and thus applies to any new physics contributing to $R_{12}$ and $R_{13}$, respecting the upper bound in Eq. (19). We conclude that in this case a non-vanishing vacuum mixing angle for the first generation neutrino is necessary. A future interesting investigation would be to determine whether the same model, but based on other types of lepton flavour violating interactions underlying the Standard Model, can represent a reasonable solution to the atmospheric and solar neutrino puzzle, or whether all such models can be excluded.

\section{Acknowledgments}

We are grateful to V. Barger and K. Whisnant for interesting discussions on their work [12]. We thank S. Nakamura for providing us with the latest results on the cross sections of the charged current reaction. We also thank T. Laval, W. Mader, A. Quadt and

G. Wilquet, for their great help in using and interpreting the results from the function minimization package MINUIT. G. M. acknowledges support from the Alexander von Humboldt Foundation, the Belgian SSTC under contract IUAP and the French Community of Belgium (ARC).

\section{References}

[1] J. N. Bahcall, CAMBRIDGE, UK: UNIV. PR. (1989), 567p.

[2] V. Barger et al., Phys. Rev. D 22 (1980) 2718.

[3] S. P. Mikheyev and A. Yu. Smirnov, Yad. Fiz. 42 (1985) 1441 [Sov. J. Nucl. Phys. 42 (1985) 913]; Nuovo Cimento 9C (1986) 17.

[4] H. A. Bethe, Phys. Rev. Lett. 56 (1986) 1305.

[5] S. P. Rosen and J. M. Gelb, Phys. Rev. D 34 (1986) 969.

[6] S. J. Parke, Phys. Rev. Lett. 57 (1986) 1275.

[7] S. M. Bilenky and S. T. Petcov, Rev. Mod. Phys. 59 (1987) 671.

[8] T. K. Kuo and J. Pantaleone, Rev. Mod. Phys. 61 (1989) 937.

[9] L. Wolfenstein, Phys. Rev. D 17 (1978) 2369.

[10] M. M. Guzzo, A. Masiero, S.T. Petcov, Phys. Lett. B 260 (1991) 154. 
[11] E. Roulet, Phys. Rev. D 44 (1991) 935.

[12] V. D. Barger, R. J. Phillips and K. Whisnant, Phys. Rev. D 44 (1991) 1629.

[13] P. I. Krastev and J. N. Bahcall, arXiv:hep-ph/9703267.

[14] S. Bergmann, M. M. Guzzo, P. C. de Holanda, P. I. Krastev and H. Nunokawa, Phys. Rev. D 62 (2000) 073001 [arXiv:hep-ph/0004049].

[15] A. M. Gago, M. M. Guzzo, P. C. de Holanda, H. Nunokawa, O. L. Peres, V. Pleitez and R. Zukanovich Funchal, Phys. Rev. D 65 (2002) 073012 [arXiv:hep-ph/0112060].

[16] M. M. Guzzo, H. Nunokawa, P. C. de Holanda and O. L. Peres, Phys. Rev. D 64 (2001) 097301 [arXiv:hep-ph/0012089].

[17] S. Bergmann, Nucl. Phys. B 515 (1998) 363 [arXiv:hep-ph/9707398].

[18] M. Guzzo, et al., Nucl. Phys. B 629 (2002) 479 [arXiv:hep-ph/0112310].

[19] J. W. Valle, arXiv:hep-ph/0205216.

[20] R. Adhikari, A. Sil and A. Raychaudhuri, Eur. Phys. J. C 25 (2002) 125 [arXiv:hep$\mathrm{ph} / 0105119]$.

[21] S. Bergmann, Y. Grossman and D. M. Pierce, Phys. Rev. D 61 (2000) 053005 [arXiv:hep-ph/9909390].

[22] M. Apollonio et al. (CHOOZ Collab.), Phys. Lett. B 466 (1999) 415 [arXiv:hepex/9907037].

[23] F. Boehm et al., Phys. Rev. D 64 (2001) 112001 [arXiv:hep-ex/0107009].

[24] V. D. Barger, arXiv:hep-ph/0102052.

[25] A. Salam and J. Strathdee, Nucl. Phys. B 87 (1975) 85.

[26] P. Fayet, Nucl. Phys. B 90 (1975) 104.

[27] For reviews see: H. E. Haber and G. L. Kane, Phys. Rep. 117 (1985) 75; H. P. Nilles, Phys. Rep. 110 (1984) 1; and S. P. Martin, arXiv:hep-ph/9709356.

[28] J. N. Bahcall et al., Astrophys. J. 555 (2001) 990; see also the J. N. Bahcall Web page: http://www.sns.ias.edu/ jnb/

[29] A. R. Junghans et al., Phys. Rev. Lett. 88 (2002) 041101 [arXiv:nucl-ex/0111014].

[30] J. N. Bahcall et al., JHEP 0204 (2002) 007.

[31] Q. R. Ahmad et al. (SNO Collab.), Phys. Rev. Lett. 87 (2001) 071301.

[32] Q. R. Ahmad et al. (SNO Collab.), Phys. Rev. Lett. 89 (2002) 011301.

[33] Q. R. Ahmad et al. (SNO Collab.), Phys. Rev. Lett. 89 (2002) 011302.

[34] G. A. McGregor (For the SNO Collaboration), arXiv:nucl-ex/0205006.

[35] H. K. Dreiner, arXiv:hep-ph/9707435.

[36] G. Bhattacharyya, Nucl. Phys. Proc. Suppl. 52A (1997) 83 [arXiv:hep-ph/9608415]. 
[37] R. Barbier et al., Report of the Group on R-parity violation (GDR-SUSY), arXiv:hepph/9810232.

[38] F. Ledroit and G. Sajot, GDR-S-008 (1998), see: http://qcd.th.u-psud.fr/GDR_SUSY/GDR_SUSY_PUBLIC/entete_note_publique

[39] B. C. Allanach, A. Dedes and H. K. Dreiner, Phys. Rev. D 60 (1999) 075014 [arXiv:hep-ph/9906209].

[40] Y. Fukuda et al. (Super-Kamiokande Collab.), Phys. Rev. Lett. 81 (1998) 1562.

[41] T. Toshito (For the Super-Kamiokande Collaboration), arXiv:hep-ex/0105023.

[42] W. C. Haxton, Phys. Rev. Lett. 57 (1986) 1271.

[43] W. C. Haxton, Phys. Rev. D 35 (1987) 2352.

[44] L. D. Landau, Phys. Z. Sowjetunion 2 (1932) 46; C. Zener, Proc. R. Soc. London A 137 (1932) 696; E. C. G. Stueckelberg, Helv. Phys. Acta 5 (1932) 369.

[45] B. T. Cleveland et al., Astrophys. J. 496 (1998) 505; Nucl. Phys. (Proc. Suppl.) B 38 (1995) 47.

[46] W. Hampel et al. (GALLEX Collab.), Phys. Lett. B 447 (1999) 127.

[47] J. N. Abdurashitov et al. (SAGE Collab.), Phys. Rev. C 60 (1999) 055801.

[48] J. N. Abdurashitov et al. (SAGE Collab.), arXiv:astro-ph/0204245.

[49] Y. Fukuda et al. (Kamiokande Collab.), Phys. Rev. Lett. 77 (1996) 1683.

[50] S. Fukuda et al. (Super-Kamiokande Collab.), Phys. Rev. Lett. 86 (2001) 5651.

[51] J. N. Bahcall et al., Phys. Rev. C 54 (1996) 411.

[52] J. N. Bahcall, Phys. Rev. C 56 (1997) 3391.

[53] S. Nakamura et al., Nucl. Phys. A 707 (2002) 561.

[54] J. N. Bahcall et al., Phys. Rev. C 55 (1997) 494.

[55] K. S. Hirata et al., Phys. Rev. D 38 (1988) 448.

[56] K. S. Hirata et al. (Kamiokande Collab.), Phys. Rev. Lett. 63 (1989) 16.

[57] K. S. Hirata et al. (Kamiokande Collab.), Phys. Rev. Lett. 65 (1990) 1297.

[58] Y. Fukuda et al. (Super-Kamiokande Collab.), Phys. Rev. Lett. 82 (1999) 2430.

[59] M. Nakahata et al. (Super-Kamiokande Collab.), Nucl. Instrum. Methods Phys. Res. Sect. A 421 (1999) 113.

[60] B. Faid, G. L. Fogli, E. Lisi and D. Montanino, Phys. Rev. D 55 (1997) 1353 [arXiv:hep-ph/9608311].

[61] J. N. Bahcall, P. I. Krastev and A. Y. Smirnov, Phys. Rev. D 58 (1998) 096016 [arXiv:hep-ph/9807216].

[62] M. C. Gonzalez-Garcia et al., Nucl. Phys. B 573 (2000) 3.

[63] G. L. Fogli and E. Lisi, Astropart. Phys. 3 (1995) 185. 
[64] M. M. Guzzo and H. Nunokawa, Astropart. Phys. 12 (1999) 87.

[65] J. N. Bahcall and R. Ulrich, Rev. Mod. Phys. 60 (1988) 297.

[66] J. N. Bahcall and M. H. Pinsonneault, Rev. Mod. Phys. 64 (1992) 885.

[67] J. N. Bahcall and M. H. Pinsonneault, Rev. Mod. Phys. 67 (1995) 781.

[68] J. N. Bahcall et al., Phys. Lett. B 433 (1998) 1.

[69] S. Nakamura et al., Phys. Rev. C 63 (2001) 034617.

[70] H. K. Dreiner and G. G. Ross, Nucl. Phys. B 365 (1991) 597.

[71] V. D. Barger, G. F. Giudice and T. Han, Phys. Rev. D 40 (1989) 2987.

[72] D. Choudhury and P. Roy, Phys. Lett. B 378 (1996) 153.

[73] ALEPH Collaboration, "Search for Supersymmetric Particles with R-Parity Violating Decays in $e^{+} e^{-}$Collisions at sqrt(s) up to 209GeV", ALEPH/2002-016, CONF/2002005, ABS276, Contributed paper to ICHEP 2002.

[74] J. Ellis et al., Mod. Phys. Lett. A 10 (1995) 1583.

[75] R. N. Mohapatra, Phys. Rev. D 34 (1986) 3457.

[76] M. Hirsch et al., Phys. Rev. Lett. 75 (1995) 17; Phys. Rev. D 53 (1996) 1329.

[77] G. F. Giudice and R. Rattazzi, Phys. Rep. 322 (1999) 421.

[78] J. E. Kim, P. Ko and D. G. Lee, Phys. Rev. D 56 (1997) 100 [arXiv:hep-ph/9701381]. 